\title{
基于混沌交织的比特交织编码调制及迭代译码 系统性能分析
}

\author{
邹雪兰 ${ }^{(1 *}$ ，刘伟彦 ${ }^{(2)}$, 㹃广增 ${ }^{(3)}$ \\ (1) 浙江传媒学院电子信息系, 杭州 310018 \\ (2) 江苏城市职业学院信息工程系, 南京 210036 \\ (3) 南京邮电大学通信与信息工程学院, 南京 210003 \\ * 通信作者. E-mail: zxl_wn@163.com
}

收稿日期: 2008-05-13; 接受日期: 2009-04-14

国家自然科学基金 (批准号: 61003088)、浙江省教育厅科研项目 (批准号: Y200906484) 和浙江省自然科学基金 (批准号: Y1100189) 资助

\begin{abstract}
摘要 比特交织编码调制及迭代译码 (BICM-ID) 是适合下一代移动通信系统的一种高效数据传输 方式. 交织器的设计是影响 BICM-ID 系统卓越性能的一个关键因素. 为了进一步提高 BICM-ID 系统 性能, 本文研究了混沌理论在交织器设计中的应用, 提出一种新的混沌交织算法, 与伪随机交织器相 比该方案具有更低的系统时延和更高的传输效率. 加性 Gauss 白噪声信道和 Rayleigh 衰落信道中的 性能仿真结果表明, 本文所提出的混沌交织器比伪随机交织器的性能要好, 在 BICM-ID 系统中具有 卓越的性能.
\end{abstract}

关键词 混沌 交织 比特交织编码调制 迭代译码

\section{1 引言}

为了满足不断增长的用户数和各种业务需求, 移动通信正朝着下一代移动通信系统发展, 其特 点是大容量、高速数据传输、支持多媒体业务. 比特交织编码调制及迭代译码 (bit-interleaved coded modulation with iterative decoding, BICM-ID) 是一种带宽有效的传输方案, 它由编码器、比特交织器 和调制器级联而成 ${ }^{[1]}$. BICM-ID 具有结构简单、实现灵活、高效传输的优点, 并且在加性 Gauss 白 噪声 (additive white Gaussian noise, AWGN) 信道和衰落信道中都具有良好的抗噪声性能, 能满足下 一代移动通信系统需要. Turbo 译码需要两个软输入软输出 (SISO) 译码器, 而 BICM-ID 只需要一个 SISO 译码器, 因此 BICM-ID 比 Turbo 码系统实现复杂度低. 同时, BICM-ID 结构灵活, 易于与其他 通信技术如 MIMO 检测 ${ }^{[2]} 、 \mathrm{OFDM}^{[3]}$ 技术相结合, 具有广泛的应用前景.

混沌理论的提出引发了人们对其应用研究的兴趣, 混沌的基本特性是对初值和系统参数极其敏感, 利用这个性质很容易产生大量不相关、可再生的随机信号. 混沌技术已经被大量地应用到信号处理和

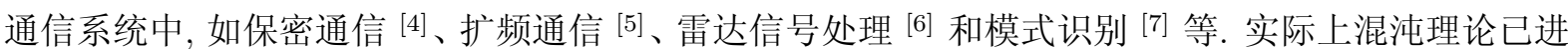




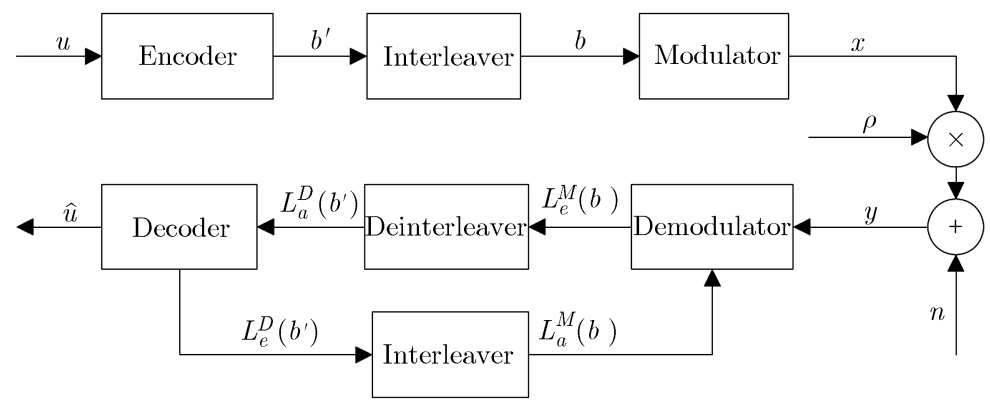

图 1 BICM-ID 系统模型

入到数字通信系统的各个领域, 包括信源编码技术、加密技术、扩频技术和调制技术等. 在现代数字 通信系统中信道编码技术是提高系统抗噪声性能的一项有效措施, 然而在纠错编码领域, 混沌的应用 报道不多.

BICM-ID 系统性能卓越的关键在于比特交织和迭代译码, 其中比特交织器能给编码系统带来分 集增益, 一般采用伪随机交织器 ${ }^{[1]}$. 本文探讨混沌技术在信道编码中的应用, 重点研究混沌交织器的 设计, 以进一步提高 BICM-ID 系统性能. 文章内容安排如下: 第 2 部分介绍 BICM-ID 系统及交织技 术相关背景; 第 3 部分给出本文提出的交织算法; 第 4 部分对混沌交织器和传统的伪随机交织器在 BICM-ID 系统中的性能进行分析和仿真比较, AWGN 和衰落信道中的仿真结果表明, 本文提出的交 织算法性能优于伪随机交织器. 第 4 部分还研究了新交织器的设计参数对系统性能的影响. 最后总结 全文.

\section{BICM-ID 系统模型}

本文研究的 BICM-ID 系统如图 1 所示. 信息序列 $\mathrm{u}$ 编码后送入交织器进行比特交织. 需要指出的 是, BICM-ID 的编码器没有任何限制, 可以是卷积码或者分组码, 也可以是 Turbo 码, 采用 Turbo 码编 码器会增加接收机的译码复杂度但能改善系统性能 ${ }^{[8]}$. 本文研究基于卷积编码的 BICM-ID 系统. 经 过比特交织得到新的序列, 并把该序列分成每 $m$ 个比特一组的子序列 $b=\left(b_{0}, b_{1}, \ldots, b_{m-1}\right)$, 调制器根 据映射规则把子序列 $b$ 映射成 M-ary 星座图中的一个符号 $x=\lambda(b)$, 其中 $\lambda$ 是映射规则, $m=\log _{2} M$.

假设信道模型为 $y=\rho x+n$, 其中 $\rho$ 是信道的衰落因子, 对频率非选择性衰落信道服从 Rayleigh 衰落分布且 $E\left(\rho^{2}\right)=1$; 对 AWGN 信道有 $\rho=1 . n$ 是单边噪声功率谱密度为 $N_{0}$ 的复 Gauss 噪声信号. 假设接收机能获知理想的信道状态信息, 即接收机可以无误差地估计 $\rho$.

接收机采用迭代译码, 在解调器和译码器之间不断地互相交换软信息从而提高对收到信号的判决 精度. 解调器的输入是接收信号 $y$ 和先验信息 $L_{a}^{M}(b)$, 输出外赋信息 $L_{e}^{M}(b)$. 外赋信息 $L_{e}^{M}(b)$ 经过解 交织后作为译码器的先验信息送入 SISO 译码器处理得到编码比特的外赋信息 ${ }^{[9,10]}$. 外赋信息 $L_{e}^{D}\left(b^{\prime}\right)$ 被交织后得到 $L_{a}^{M}(b)$ 作为解调器的先验概率信息进行下一轮迭代处理. 在第一次迭代处理时, 先验信 息 $L_{a}^{M}(b)$ 设为 0 ; 由于译码器没有使用信息比特的先验信息, 信息比特的外赋信息就是总的后验概率, 最后一次迭代译码时译码器对信息比特的外赋信息做硬判决得到信息比特的估计值 $\hat{u}$.

交织是通信系统中广泛应用的一种信号处理技术. 交织器是一个单输入单输出的设备, 其输入、 输出序列具有相同的元素符号, 但是各个符号在输入序列和输出序列中的排列顺序不同. 交织可由函 
数表示为

$$
\pi: Z \rightarrow Z \text {. }
$$

交织实际上是对整数 $Z$ 做置换变换. 交织通常可分为两种: 分组交织和卷积交织. BICM-ID 系统中采 用的伪随机交织器是在传统的分组交织器基础上演化而来的伪随机分组交织器. 把随机噪声序列以某 种方式排序得到的置换序列作为交织序列得到伪随机交织器 [11]. 通常, 随机交织器的性能要优于传 统的分组交织器. 本文的噪声矢量是通过 Matlab 函数产生均匀分布的噪声源得到.

\section{3 混沌交织算法}

从图 1 可以看出交织器是 BICM-ID 系统的一个重要组成部分. 本节我们研究如何结合混沌技术 来设计交织器以优化 BICM-ID 系统性能. 受伪随机交织器的工作原理启发, 可把混沌函数生成的混 沌序列作为交织序列从而得到混沌交织器. 本文重点研究一维混沌映射, Logistic 映射是一个典型的 一维混沌映射, 该映射在许多研究领域得到了广泛应用, 本文以 Logistic 映射为例讨论混沌交织器的 设计. Logistic 映射定义如下 ${ }^{[12]}$ :

$$
X_{n+1}=\mu X_{n}\left(1-X_{n}\right), \quad 0<x_{n}<1, \quad 0<\mu<4, \quad n=0,1,2,3, \ldots
$$

式中 $\mu$ 是分岔参数 (bifurcation parameter), $\mu$ 取值不同, Logistic 映射呈现出明显的周期性或混沌行 为. 当 $\mu$ 接近 4 的时候, Logistic 系统进入混沌状态, 所产生的序列是混沌序列.

根据伪随机交织器的产生原理, 把式 (2) 产生的混沌序列代替伪随机交织器中的噪声序列, 对该 序列进行排序, 所得到的置换序列作为交织序列就得到混沌交织器. 混沌序列具有各态历经性、随机 性, 同时又具有确定性, 因此混沌交织器性能将优于伪随机交织器. 为了简化交织器的实现复杂度, 改 善其性能, 有很多技术可以优化交织器的设计. 我们在混沌交织器的设计中引入模 2 特性. 模 2 交织 器 (也称为奇偶交织器) 的概念是 Barbulescu 等 [13] 首次提出的. 模 2 特性要求比特的位置在交织前 后具有相同的模 2 值, 即编码器输出的码序列中居于偶数位置上的比特只能被映射到交织序列的偶数 位置上, 而交织前居于码序列中奇数位置上的比特还处于交织序列的奇数位置上. 文献 [13] 指出, 在 码率为 $1 / 2$ 的 Turbo 码系统中具有模 2 特性的交织器能带来系统性能增益, 因为该特性使得每个信息 比特有且只有一个校验比特保留, 这样码的纠错能力在所有信息比特之间均匀分配. 结合混沌映射和 模 2 特性, 本文提出一种新的交织算法, 称该交织器为混沌一模 2 交织器 (chaotic-mod2-interleaver), 具体设计步骤如下:

1) 根据式 (2) 产生一个长度为 $L$ 的混沌序列 $\alpha$, 其中 $L$ 是交织长度. 对序列 $\alpha$ 进行排序得到索 引序列 $s, s$ 与排序后的序列 $\tilde{\alpha}$ 之间的关系如下:

$$
\tilde{\alpha}(k)=\alpha(s(k)), \quad k=1,2, \ldots, L .
$$

2) 使序列 $\mathrm{s}$ 中的元素满足模 2 特性, 得到新的序列 $z, z$ 中元素满足

$$
z_{i}=\left\{\begin{array}{ll}
s_{j}, & \text { 当 } \bmod \left(s_{j}, 2\right)=1, \bmod (i, 2)=1, \\
s_{j}, & \text { 当 } \bmod \left(s_{j}, 2\right)=0, \bmod (i, 2)=0,
\end{array} \quad i=1, \ldots, L, j=1, \ldots, L,\right.
$$

序列 $z$ 就是混沌一模 2 交织器的交织序列. 
对码率为 $1 / 2$ 的递归系统卷积码来说, 模 2 特性确保所有的信息比特在交织后有且只有一个校 验比特, 因此码比特功率在所有比特之间均匀分布. 如果没有模 2 特性, 信息比特和校验比特在交织 后的序列中可能不会均匀分布, 在这种情况下, 有些干扰就会影响那些后面没有校验比特的信息比特, 译码器对这些比特译码时性能可能下降. 因此, 模 2 特性的引入使得混沌一模 2 交织器的性能比混沌 交织器的要好. 混沌一模 2 交织器也可用于其他码率的卷积码系统. 对采用码率为 $2 / 3$ 的递归系统卷 积码的 BICM-ID 系统性能仿真表明混沌一模 2 交织器的性能略差于伪随机交织器的性能. 对 $2 / 3$ 码 率的递归系统卷积码来说, 编码器输出序列中每 2 个信息比特后有 1 个校验比特, 而模 2 特性不能保 证编码功率在所有信息比特中均匀分配. 例如, 设交织器的输入为

$$
\left(c_{11}, c_{12}, c_{13}, c_{21}, c_{22}, c_{23}, c_{31}, c_{32}, c_{33}\right),
$$

而混沌一模 2 交织器的输出是

$$
\left(c_{11}, c_{12}, c_{22}, c_{21}, c_{31}, c_{32}, c_{13}, c_{23}, c_{33}\right),
$$

式中 $c_{i 1}, c_{i 2}(i=1,2,3)$ 表示信息比特, $c_{i 3}(i=1,2,3)$ 表示校验比特. 为了改善系统性能, 我们提出具有 模 3 特性的交织器 (混沌一模 3 交织器) 解决方案. 混沌一模 3 交织器的算法步骤与混沌一模 2 交织器 的类似, 不同的是在步骤 2 中要求序列满足模 3 特性. 因此, 在混沌交织器中引入模 $k(k=2,3,4, \ldots)$ 性质可以满足不同码率系统的要求. 混沌技术也可用于改善其他交织器的性能, 如文献 [14] 引入混沌 技术明显改善了黄金分割交织器在 BICM-ID 系统中的性能. 可见混沌技术为改进交织器的性能和实 现打开了新思路.

混沌交织器具有传送参数少的优点, 而伪随机交织器需要把整个交织序列从发送端传递到接收端, 因此混沌交织器可节省信道资源, 同时减少传输过程中发生的数据差错概率. 混沌一模 2 交织器继承 了混沌交织器的优点. 下面将通过仿真来验证本文所提出算法的有效性, 其中 random int 指伪随机交 织器, chaotic int 表示混沌交织器, 而 chaotic-mod2 int 表示混沌一模 2 交织器, chaotic-mod3 int 表示 混沌一模 3 交织器.

\section{4 性能分析}

\section{1 相关系数分析}

文献 [15] 提出用相关系数分析交织器的性能. 假设交织器的输入序列是 $s_{0}^{i}, s_{1}^{i}, \ldots, s_{N-2}^{i}, s_{N-1}^{i}$, 输 出序列为 $s_{0}^{o}, s_{1}^{o}, \ldots, s_{N-2}^{o}, s_{N-1}^{o}$, 则交织器的相关系数定义为 [15]

$$
R=\sum_{k=0}^{N-1}\left(2 s_{k}^{i}-1\right)\left(2 s_{k}^{o}-1\right) .
$$

式 (7) 是交织器的输入序列和输出序列之间的相关系数. 根据概率论, 如果输入序列与输出序列互相 独立, 相应的相关系数为 0 . 然而实际的输入、输出序列之间总存在一定的联系. 在 Turbo 码系统中相 关系数低的交织器具有更好的性能 ${ }^{[11,15]}$. 表 1 给出了文中涉及的 3 种交织器在不同交织长度条件下 的相关系数. 表 1 表明, 相关系数随着交织长度的增加而减小, 这与增加交织长度能提高系统性能的 结论一致 ${ }^{[1]}$. 从相关系数分析来看, 在给定交织长度的前提下, 混沌一模 2 交织器的相关系数比混沌 交织器和伪随机交织器的要高. 究其原因, 前面提到交织器的模 2 特性要求比特的位置在交织前后具 
表 1 不同交织算法的相关系数

\begin{tabular}{cccc}
\hline Length & Random int & Chaotic int & Chaotic-mod2 int \\
\hline 600 & $1.680000 \mathrm{e}-003$ & $1.738333 \mathrm{e}-003$ & $3.490000 \mathrm{e}-003$ \\
1000 & $1.021000 \mathrm{e}-003$ & $1.022000 \mathrm{e}-003$ & $2.033000 \mathrm{e}-003$ \\
2004 & $5.294411 \mathrm{e}-004$ & $5.434132 \mathrm{e}-004$ & $1.031437 \mathrm{e}-003$ \\
6000 & $1.700000 \mathrm{e}-004$ & $1.670000 \mathrm{e}-004$ & $3.375000 \mathrm{e}-004$ \\
\hline
\end{tabular}

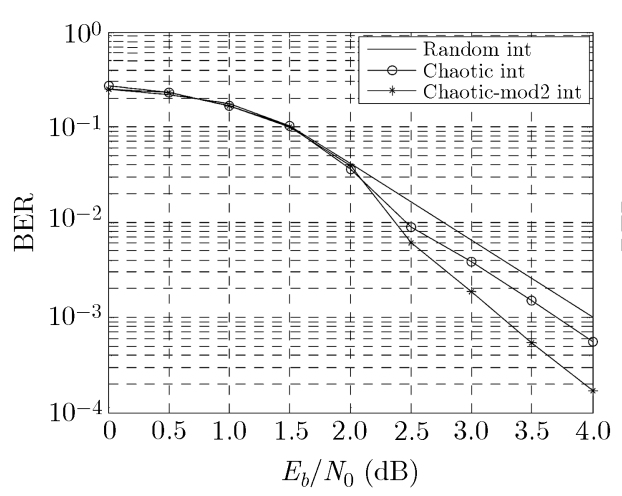

(a) 交织长度为 600 bits

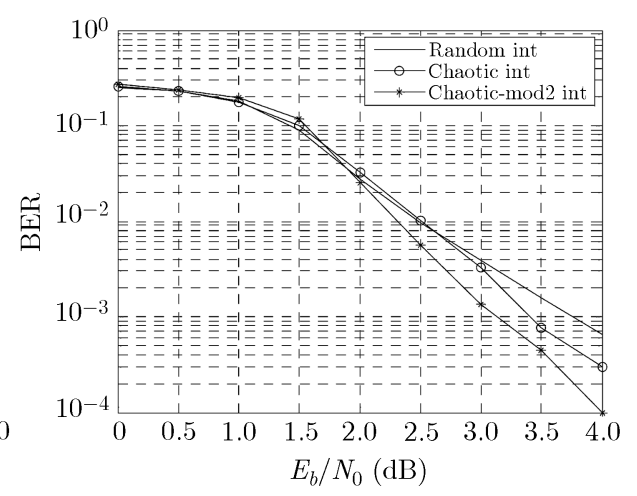

(b) 交织长度为 2400 bits

图 2 AWGN 信道中基于混沌的交织器与伪随机交织器的性能比较曲线

有相同的模 2 值, 该性质确保编码功率在所有比特之间均匀分配, 但它降低了交织输出序列的随机性, 从而增加了混沌一模 2 交织器的相关系数. BICM-ID 系统中的交织器能为编码系统带来分集增益, 而 迭代译码系统是一个复杂的非线性系统, 仅从相关系数的细小差异很难评价交织器的实际性能, 因此 我们通过性能仿真来研究交织器的设计对 BICM-ID 系统的性能影响.

\section{2 仿真结果}

本节我们通过仿真分析交织器的设计对 BICM-ID 系统性能的影响, 系统参数设置如下: 编码器 采用 $1 / 2$ 码率、 4 状态递归系统卷积码, 8-PSK 调制, 映射方式为 SP (set partitioning) 映射; 接收端采 用 Log-MAP 译码算法, 迭代译码次数为 8 次. 除非比较交织器的设计参数对 BICM-ID 系统性能影 响, 分岔参数取值均为 3.7. 图 2 是 AWGN 信道中混沌交织器、混沌一模 2 交织器与伪随机交织器的 性能比较曲线, 交织长度分别是 600 和 2004 bits. 图 2 表明混沌交织器比伪随机交织器的性能要好, 而混沌一模 2 交织器与混沌交织器相比又有性能改善. 图 2(a) 表明, 在 $B E R=10^{-3}$ 时, 混沌一模 2 交织器比伪随机交织器有 $0.7 \mathrm{~dB}$ 的性能增益, 混沌交织器比伪随机交织器有 $0.3 \mathrm{~dB}$ 性能增益.

图 3 是 Rayleigh 衰落信道中交织器的性能比较曲线. 图 3(b) 表明, 在交织长度为 $2004 \mathrm{bits}$ 和 $\mathrm{BER}=10^{-4}$ 时, 混沌一模 2 交织器比伪随机交织器大约有 $2 \mathrm{~dB}$ 的性能增益, 比混沌交织器有大约 $1 \mathrm{~dB}$ 的性能增益. 图 3 表明在 Rayleigh 衰落信道中混沌一模 2 交织器性能最好, 混沌交织器比伪随 机交织器的性能好. 从图 2 和 3 可以看出, 交织长度为 600 bits 的混沌一模 2 交织器的性能比交织长 度为 2004 bits 的伪随机交织器的好, 这说明当提供相同的 BER 性能时, 混沌一模 2 交织器所需要的 交织长度远小于伪随机交织器的交织长度. 交织长度越大, 系统需要的处理时延越大, 混沌一模 2 交 织器具有更低的处理时延, 从而能减少系统处理时延. 仿真结果表明, 模 2 特性的引入虽然增加了一 


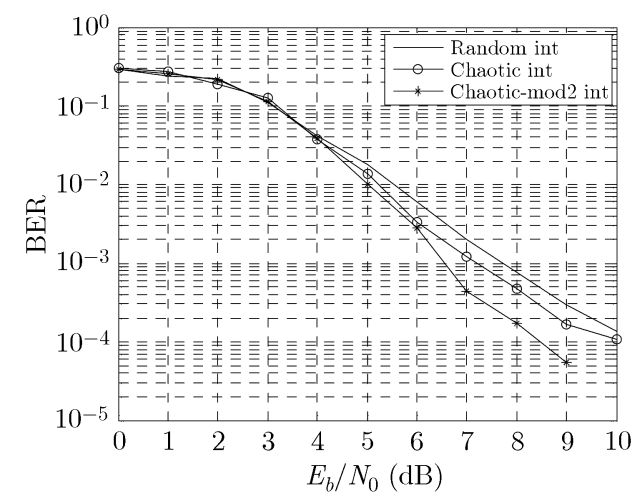

(a) 交织长度600 bits

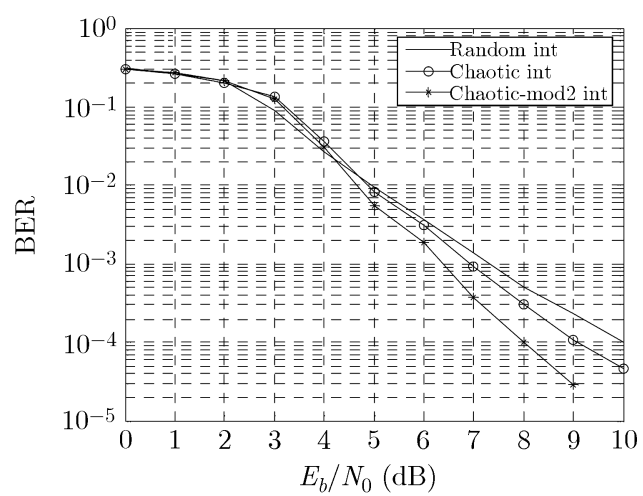

(b) 交织长度2004 bits

图 3 Rayleigh 衰落信道中基于混沌的交织器与伪随机交织器的性能比较曲线

定的计算复杂度 (为混沌序列增加模 2 计算), 但是大大提高了混沌交织器的性能, 并且混沌一模 2 交 织器具有更低的处理时延, 在实际应用中具有优势.

图 4 是 AWGN 信道和 Rayleigh 衰落信道混沌一模 2 交织器、混沌一模 3 交织器与伪随机交织 器的性能比较结果. 编码器是 $2 / 3$ 码率、 4 状态递归系统卷积码, 混沌一模 3 交织器与伪随机交织器 的交织长度是 $1503 \mathrm{bits}$, 为了保证模 2 特性, 混沌一模 2 交织器的长度设为 $1506 \mathrm{bits}$, 其他仿真参数 同上. $2 / 3$ 码率卷积码由 $1 / 2$ 码率递归系统卷积码通过下面的删余矩阵删余得到

$$
P=\left[\begin{array}{ll}
1 & 1 \\
1 & 0
\end{array}\right] .
$$

如果删余前的码组为

$$
\left(c_{11}, c_{12}, c_{21}, c_{22}, c_{31}, c_{32}, c_{41}, c_{42}, \ldots\right)
$$

则删余后得到

$$
\left(c_{11}, c_{12}, c_{21}, E, c_{31}, c_{32}, c_{41}, E, \ldots\right) .
$$

$c_{i 1}(i=1,2,3, \ldots)$ 表示信息比特, 而 $c_{i 2}(i=1,2,3, \ldots)$ 是校验比特, $E$ 表示被删余的比特, 在数据传输 中该比特不会进入信道传送. 当系统采用 $2 / 3$ 码率递归系统卷积码时, 在 AWGN 信道和 Rayleigh 衰 落信道中, 混沌一模 3 交织器在 3 种交织算法中性能最好, 而混沌一模 2 交织器要略比伪随机交织 器的性能差. 图 4(a) 表明, 在在 AWGN 信道中, 当 BER $=10^{-3}$ 时, 混沌一模 3 交织器比混沌一模 2 交织器有 $0.9 \mathrm{~dB}$ 的性能增益, 比伪随机交织器有 $0.6 \mathrm{~dB}$ 的性能增益. 在 Rayleigh 衰落信道, 图 4(b) 表明, 在 $\mathrm{BER}=10^{-3}$ 时, 混沌一模 3 交织器性能优于混沌一模 2 交织器大约 $1.3 \mathrm{~dB}$, 而伪随机交织 器相对混沌一模 2 交织器的性能增益是 $0.7 \mathrm{~dB}$. 当信噪比较低的时候 (AWGN 信道中 $\mathrm{SNR}<4.5 \mathrm{~dB}$, Rayleigh 衰落信道 $\mathrm{SNR}<6 \mathrm{~dB}) 3$ 种交织器的性能差别不大, 误比特率都比较大. 混沌一模 2 交织器也 适用编码器为 $2 / 3$ 码率卷积码的 BICM-ID 系统, 由于混沌一模 3 交织器比伪随机交织器有明显的性 能改善因此采用混沌一模 3 交织器更好.

显然 Logistic 映射的分岔参数是影响混沌交织器性能的重要参数. 混沌系统是非线性系统, 难以 直接给出分岔参数对混沌交织器性能影响的闭合表达式. Logistic 映射随着分岔参数 $\mu$ 取值不同, 呈 现出周期性或混沌行为. 下面我们对 $\mu$ 的 3 个典型取值即 $\mu=3.5, \mu=3.7$ 和 $\mu=3.9$ 通过仿真来分 析分岔参数对混沌一模 2 交织器的性能影响. 当 $\mu=3.5$ 时 Logistic 映射进入周期状态, 而当 $\mu=3.7$ 


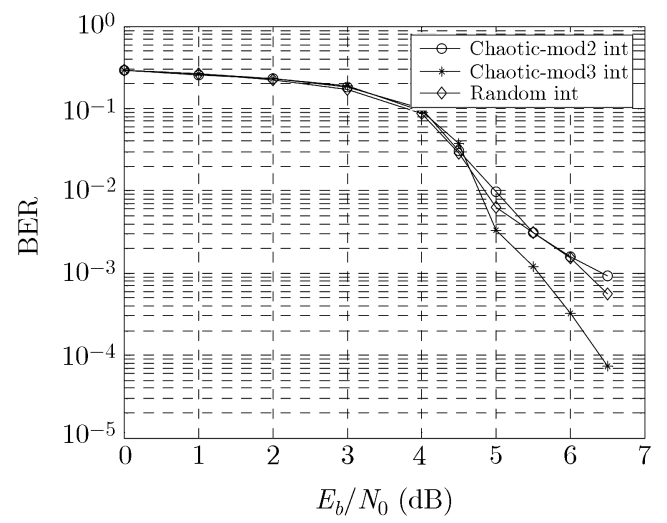

(a) AWGN信道

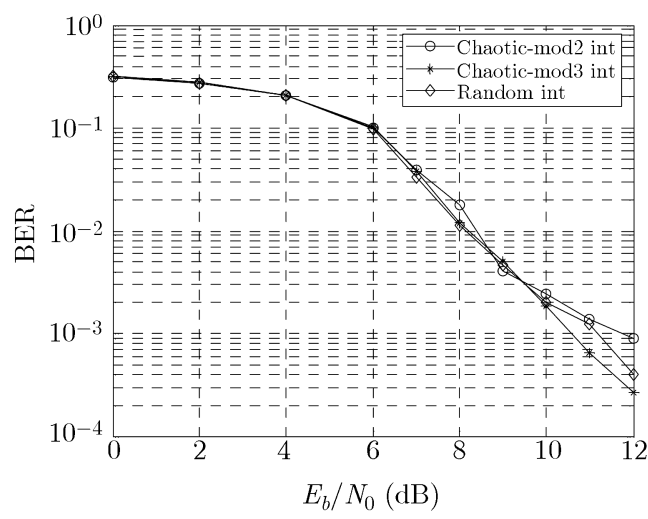

(b) Rayleigh衰落信道

图 4 交织算法对 BICM-ID 系统性能影响

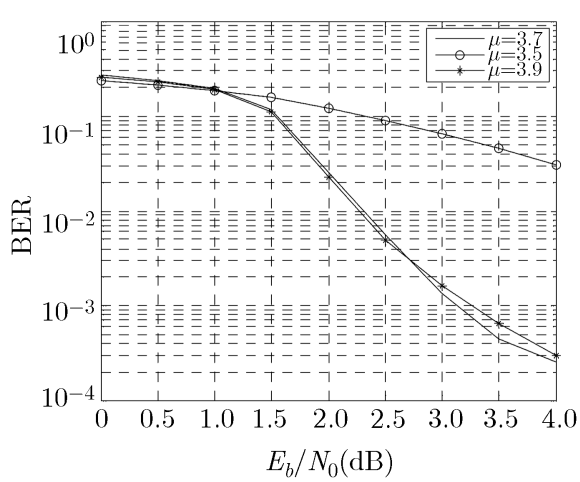

(a) AWGN信道

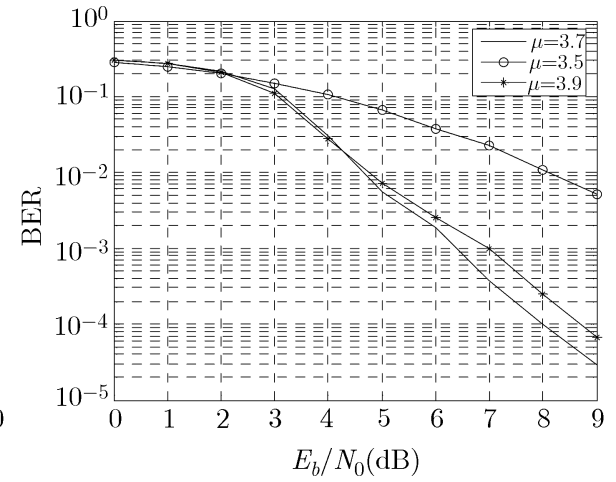

(b) Rayleigh衰落信道

图 5 分岔参数 $\mu$ 对混沌一模 2 交织器的性能影响

和 $\mu=3.9$ 时 Logistic 映射进入混沌状态 ${ }^{1)}$. 图 5 分别是在 AWGN 信道和 Rayleigh 衰落信道中, 参 数 $\mu$ 对混沌一模 2 交织器的性能影响仿真结果, 其中交织长度为 2004 bits. 可以看到, 对于混沌一模 2 交织器当 $\mu=3.5$ 时, 它的性能远不如 $\mu=3.7$ 和 $\mu=3.9$ 时的性能. 比较图 $2,3,5, \mu=3.5$ 的混沌 一模 2 交织器的性能甚至不如伪随机交织器. $\mu=3.7$ 时混沌一模 2 交织器的性能比 $\mu$ 的另外两个取 值性能要好. 这说明要使混沌一模 2 交织器具有好的性能, 要求选择合适的参数 $\mu$ 使 Logistic 映射产 生的序列是混沌序列. 在保证 Logistic 映射产生的序列是混沌序列的前提下, 本文的仿真结果表明参 数 $\mu$ 的取值对混沌一模 2 交织器的性能影响不明显.

\section{5 结论}

混沌理论在信号处理和通信系统中得到了广泛的应用. 本文研究了混沌技术在 BICM-ID 系统 中的应用, 提出一种基于混沌映射的交织算法. 与传统的伪随机交织器相比, 本文提出的交织算法在 AWGN 信道和 Rayleigh 衰落信道中都有显著的性能改善; 并且该算法具有传送参数少、系统处理时

1) Elert G. Measuring Chaos. http://hypertextbook.com/chaos/4_3 Lyapunov Exponent.htm 
延小、能降低传输中由于参数传输错误而引起的误码率等优点. 文中通过仿真研究了交织器设计参数 对 BICM-ID 系统性能的影响. 只要分岔参数的取值能保证 Logistic 映射进入混沌状态, 本文提出的混 沌交织器能给系统带来明显的性能增益. 除了本文提到的模 $k(k=2,3)$ 性质外, 还有许多其他技术可 以优化交织器的设计. 混沌技术为交织器的性能优化和实现打开了新的解决思路, 如何开发混沌理论 在数字通信系统中的应用有待人们继续深入研究.

\title{
参考文献
}

1 Li X, Chindapol A, Ritcey J A. Bit-interleaved coded modulation with iterative decoding and 8PSK signaling. IEEE Trans Commun, 2002, 50: 1250-1257

2 Nekuii M, Davidson T N. Reduced-complexity demodulation for MIMO-BICM-IDD using modified stack algorithms. IEEE ICASSP, 2007, 3: 65-68

3 Tran N H, Nguyes H H, Le-Ngoc T. Bit-interleaved coded OFDM with signal space diversity: subcarrier grouping and rotation matrix design. IEEE Trans Signal Process, 2007, 55: 1137-1149

4 Chen X, Wang Z. An observer-based chaotic synchronization scheme for time-delay secure communication systems. In: IEEE Int. Conf. on Networking, Sensing and Control. London, 2007. 209-211

5 Heideri-Bateni G, McGillem C D. A chaotic direct sequence spread spectrum communication system. IEEE Trans Commun, 1994, 42: 1524-1527

6 Leung H. Applying chaos to radar detection in an ocean environment: an experimental study. IEEE J Ocean Eng, 1995, 20: 56-64

7 Andreyev Y V, Dmitriev A S, Starkov S O. Information processing in 1-D systems with chaos. IEEE Trans Circuits Syst I, 1997, 44: 21-28

8 Abramovici I, Shamai S. On turbo encoded BICM. Annual Telecommun, 1999, 54: 225-234

9 Benedetto S, Divsalar D, Monrorsi G, et al. A soft-input soft-output APP modules for iterative decoding of concatenated codes. IEEE Commun Lett, 1997, 1: 22-24

10 Zou X, Feng G. A modified max-log-MAP algorithm for turbo decoding. J China Univ Posts Telecommun, 2002, 9: $7-12$

11 Heegard C, Wicker S B. Turbo Coding. Boston/Dordrecht/London: Kluwer Academic Publishers, 1999

12 Petitgen H, Jurgens H, Saupe D. Chaos and Fractals: New Frontiers of Science. Berlin: Spring-Verlag, 1992

13 Barbulescu A S, Pietrobon S S. Interleaver design for turbo codes. Electron Lett, 1994, 30: 2107-2108

14 Zou X, Wang M, Feng G. A new interleaver design for iteratively decoded bit-interleaved coded modulation. Int J Soft Comput, 2008, 3: 338-343

15 Fan P, Zhang J, Cao Z. A design method of block interleaver with low correlation (in Chinese). J China Instit Commun, 1998, 19: 50-54

\section{Performance of iteratively decoded bit-interleaved coded modulation with chaos based interleaving strategies}

\author{
ZOU XueLan ${ }^{1 *}$, LIU WeiYan ${ }^{2} \&$ FENG GuangZeng ${ }^{3}$ \\ 1 Department of Electronic and Information Engineering, Zhejiang Institute of Media and Communications, \\ Hangzhou 310018, China; \\ 2 Department of Information Engineering, Jiangsu City Vocation College, Nanjing 210036, China; \\ 3 College of Communication and Information Engineering, Nanjing University of Posts and Telecommunications,
}


Nanjing 210003, China

*E-mail: zxl_wn@163.com

Abstract Bit-interleaved coded modulation with iterative decoding (BICM-ID) is a bandwidth efficient transmission scheme which is well suitable for next-generation mobile communications. The interleaver design is critical to the high performance of BICM-ID. In this paper we investigate the application of the chaos theory to the interleaver design to further improve the performance of BICM-ID. A new interleaving scheme based on the chaos theory is proposed. Compared with the random interleaver, the proposed approach has the advantages including lower system delay and higher transmission efficiency. Simulation results in both additive white Gaussian noise (AWGN) channel and Rayleigh fading channel are provided, which demonstrates that the new interleaver is better than the random interleaver and exhibits excellent performance when applied to the BICM-ID system.

Keywords chaos, interleaving, bit-interleaved coded modulation (BICM), iterative decoding 